\title{
CORRIGENDUM
}

\section{High prevalence of diabetes, obesity and dyslipidemia in urban slum population of northern India}

\author{
A Misra et al \\ International Journal of Obesity (2002) 26, 1281. doi:10.1038/sj.ijo.0802030
}

Correction to: International Journal of Obesity (2001) 25, 1722-1729

Following the publication of the above paper, the author has identified errors in Table 2. The correct version of Table 2 is reproduced below.

Table 2 Profile of various measures of obesity

\begin{tabular}{|c|c|c|c|c|}
\hline \multirow[b]{2}{*}{ Measures of obesity } & \multicolumn{2}{|c|}{ Males } & \multicolumn{2}{|r|}{ Females } \\
\hline & $\mathrm{n}$ & Mean \pm s.d. & $\mathrm{n}$ & Mean \pm s.d. \\
\hline Body mass index & 165 & $20.0 \pm 4.1$ & 359 & $20.7 \pm 4.5(\mathrm{NS})$ \\
\hline \multicolumn{5}{|l|}{ Skinfolds $(\mathrm{mm})$ and ratios } \\
\hline Waist-hip ratio & 170 & $0.86 \pm 0.11$ & 362 & $1.02 \pm 0.39(\mathrm{NS})$ \\
\hline Biceps & 170 & $5.5 \pm 3.8$ & 362 & $8.3 \pm 5.4^{*}$ \\
\hline Triceps & 170 & $9.7 \pm 6.6$ & 362 & $14.8 \pm 7.8^{*}$ \\
\hline Subscapular & 170 & $13.1 \pm 7.3$ & 362 & $17.1 \pm 9.5^{*}$ \\
\hline Suprailiac & 169 & $14.3 \pm 7.5$ & 361 & $19.9 \pm 11.3^{*}$ \\
\hline$\Sigma 4$ SF (sum of four skinfolds) & 169 & $42.3 \pm 21.2$ & 361 & $60.0 \pm 30.9^{*}$ \\
\hline $\begin{array}{l}\text { Central skinfolds (sum of } \\
\text { subscapular and suprailiac skinfolds) }\end{array}$ & 169 & $27.2 \pm 13.0$ & 361 & $36.9 \pm 19.8^{*}$ \\
\hline Peripheral skinfolds (sum of triceps and biceps skinfolds) & 170 & $15.2 \pm 9.6$ & 362 & $23.1 \pm 12.6^{*}$ \\
\hline Central-peripheral skinfold ratio & 169 & $2.01 \pm 0.69$ & 361 & $1.67 \pm 0.59^{*}$ \\
\hline SS/TR (Subscapular-triceps skinfold ratio) & 170 & $1.54 \pm 0.64$ & 362 & $1.23 \pm 0.7^{*}$ \\
\hline Body fat (\%) & 169 & $17.5 \pm 6.2$ & 361 & $28.4 \pm 7.6^{*}$ \\
\hline
\end{tabular}

NS, statistically not significant; ${ }^{*} P<0.001$. 